\title{
Aplicação R-Otimizada no Zulliger: Evidências de Validade com Pacientes Depressivos
}

\author{
André Pereira Gonçalves ${ }^{1}$, Ana Carolina Zuanazzi, Anna Elisa de Villemor-Amaral \\ Universidade São Francisco, Campinas-SP, Brasil
}

\section{RESUMO}

A proposta de uso do Zulliger controlando o número de respostas (R-Otimizado) é inédita e, com isso, é necessária a verificação empírica de suas evidências de validade, objetivo central deste estudo. Levando em conta as similaridades dos métodos de Zulliger e Rorschach, procurou-se evidências de validade para o Zulliger, aplicação R-otimizada, por meio da concordância com Rorschach nas variáveis relacionadas à depressão. Para a amostra, foram recrutados 39 sujeitos, com diagnóstico primário de depressão e que apresentaram sintomatologia depressiva na Escala Baptista de Depressão. Foram administrados os métodos de Zulliger e Rorschach de forma alternada para controle do efeito de um instrumento sobre o outro. Para as análises, foram selecionadas as variáveis relacionadas à depressão, segundo literatura prévia. Os resultados demonstraram evidências iniciais de validade para algumas variáveis relacionadas à depressão, melhorando a correlação entre elas, quando comparado a estudos anteriores que não utilizaram o Zulliger aplicação R-otimizada. Palavras-chave: avaliação psicológica; depressão; psicopatologia; técnicas projetivas; Rorschach.

\section{ABSTRACT - R-Optimized Administration in Zulliger: Validity Evidence for Depressive Patients}

The proposal of administrating Zulliger controlling the number of responses (R-Optimized) was never tried and it is necessary to verify empirically its validity evidences, our aim in this study. For this, 39 subjects were recruited, with a primary diagnosis of depression and minimal symptomatology within the light classification. The Baptist Depression Scale (EBADEP-A) was used to verify the symptomatologic level and, afterwards, the Zulliger and Rorschach tests were applied alternately to control the effect of one instrument on the other. For the analyzes, the variables related to depression of both instruments were selected according to previous literature. The results demonstrated initial evidence of validity for some variables related to depression, improving the correlation between them, when compared to previous studies in which the number of responses in Zulliger was not controlled. Further studies on Zulliger using R-optimized application are needed to confirm these findings.

Keywords: psychological assessment; depression; psychopathology; projective techniques; Rorschach.

\section{RESUMEN - Aplicación R-optimizado en Zulliger: Evidencias de Validez con Pacientes Depresivos}

La propuesta de la utilización de Zulliger controlando el número de respuestas (R-Optimizado) es de carácter novedoso, por lo tanto, es necesario la verificación empírica para comprobar sus evidencias de validez, siendo este, el objetivo central del estudio. Teniendo en cuenta las similitudes de los métodos de Zulliger y Rorschach, se buscó evidencias de validez para el Zulliger aplicación R-optimizada por medio de la concordancia con Rorschach en las variables relacionadas a la depresión. Para la muestra, fueron reclutados 39 sujetos, con diagnóstico primario de depresión y que presentaron sintomatología depresiva en la Escala Baptista de Depresión. Se administraron los Métodos de Zulliger y Rorschach de forma alternada para controlar el efecto de un instrumento sobre el otro. Para los análisis, se seleccionaron las variables relacionadas a la depresión, según literatura previa. Los resultados demostraron evidencias iniciales de validez para algunas variables relacionadas a la depresión, mejorando la correlación entre las mismas, en comparación con estudios anteriores que no utilizaron el Zulliger aplicación R-optimizado.

Palabras clave: evaluación psicológica; depresión; la psicopatología; técnicas proyectivas; Rorschach.

A depressão é um dos transtornos mentais mais frequentes em todo o mundo, afetando mais de 300 milhões de pessoas, segundo dados da Organização Mundial de Saúde (OMS, 2017). Apesar dos critérios diagnósticos bem definidos tanto no Manual Diagnóstico e Estatístico de Transtornos Mentais - 5a edição (APA, 2014) quanto na CID-10 (OMS, 1993), na prática clínica, a avaliação psicológica dessa psicopatologia é uma tarefa complexa devido a seus sintomas também se manifestarem em outras psicopatologias. Com isso, torna-se necessária uma investigação cuidadosa por parte do psicólogo e demais profissionais da saúde para minimizar as chances de um diagnóstico equivocado que possa gerar danos na vida da pessoa (Baptista, 2012).

Como uma possibilidade de auxiliar no processo diagnóstico, a avaliação multimétodo há tempos tem-se 
mostrada muito eficiente para esse fim devido às possiblidades de apreender um fenômeno por vários ângulos diferentes (Campbell \& Fiske, 1959; Mihura, 2012; Mihura \& Graceffo, 2014). Nessa perspectiva, o uso de instrumentos psicológicos de diferentes tipos, como escalas, entrevistas e as técnicas projetivas, auxilia nesse processo de coleta de informações sobre o sujeito. O uso de técnicas projetivas é importante no contexto da avaliação, pois acessam informações em níveis diferentes das escalas, o que proporciona mais informações sobre o mesmo construto (Meyer et al., 2017).

Dentre os instrumentos projetivos, têm-se os testes de manchas de tinta, sendo o das manchas de tinta de Rorschach e Zulliger os mais pesquisados no contexto brasileiro (Grazziotin \& Scortegagna, 2016; Pianowski, Villemor-Amaral, Yazigi, Nascimento, \& Miguel, 2017). Ambos guardam como características comuns o tipo de estímulos - manchas de tintas semiestruturadas e simétricas - bem como as instruções e o padrão de análise das variáveis produzidas. Por meio dessa tarefa, é possível observar o modo de percepção do sujeito e, assim, avaliar formas de seu funcionamento psíquico que fornecem informações sobre os estilos de tomada de decisões e maneiras de pensar, agir e sentir, evidenciados tanto no Zulliger (Villemor-Amaral \& Cardoso, 2012; VillemorAmaral \& Primi, 2009) quanto no Rorschach (Meyer, Viglione, Mihura, Erard, \& Erdberg, 2017; Weiner \& Green, 2017).

No Brasil, para uso profissional, há dois sistemas disponíveis para administração, codificação e interpretação do Zulliger: sistema Klopfer (Vaz \& Alchieri, 2016) e sistema Compreensivo (VillemorAmaral \& Primi, 2009), estudado também em outros países (Mahmood, 1990; Mattlar, Sandahl, Lindberg, \& Lehtinen, 1990; Zdunic, 1999). Este último sistema possibilita a avaliação de adultos e crianças (Biasi \& Villemor-Amaral, 2016) e possui estudos com grupo de depressivos (Grazziotin \& Scortegagna, 2016). Para o Rorschach, há cinco sistemas vigentes, dentre eles o sistema Compreensivo (SC, Exner \& Sendín, 1999) e o sistema de Avaliação por Performance no Rorschach (R-PAS, Meyer et al., 2017), que basearam a construção metodológica do presente estudo.

Estudos sobre similaridades e diferenças das duas técnicas vêm sendo realizados por pesquisadores da área (Cárpio \& Lugón 2011; Villemor-Amaral \& Cardoso, 2012; Villemor-Amaral, Pianowski, \& Carvalho, 2016). Algumas variáveis comuns entre as duas técnicas apresentaram correlações fracas a fortes, como a proporção no número de reposta $(\mathrm{R} ; r=0,59)$, movimento humano $(\mathrm{M} ; r=0,51)$, respostas par $(2 ; r=0,50)$, conteúdo animal $(\mathrm{A} ; r=0,45)$, espaço em branco $(\mathrm{S} ; r=0,18)$, respostas de cor acromática com influência de forma (FC'; $r=0,19)$ e conteúdos de Arte (Art; $r=0,19)$ (Cárpio \& Lugón, 2011). Em relação aos cálculos, foram encontradas correlações referentes ao escore WSumC $(r=0,34)$ e aos tipos de vivência (EB) extratensivo $(r=0,312)$, evitativo $(r=0,519)$ e introversivo $(r=0,153)$ (Villemor-Amaral \& Cardoso, 2012).

A pesquisa mais recente encontrada sobre a similaridade das técnicas foi realizada por Villemor-Amaral et al. (2016). Esse estudo teve como objetivo verificar o alcance e as limitações dos estímulos de indicadores de cor (C), movimento humano $(\mathrm{M})$ e número de resposta (R) no Zulliger usando o Rorschach como referência. A amostra contou com 51 pessoas, dividida em dois grupos: concordante, que corresponde ao grupo que apresentou concordância entre os tipos de vivências identificados no Zulliger e Rorschach $(n=24)$, e discordante, que apresentaram resultados diferentes entre os tipos de experiências no verificados em ambos os testes $(n=27)$.

Os autores verificaram, em relação à amostra total $(N=51)$, baixas magnitudes de diferença entre os dois métodos, tanto nos estímulos acromáticos $(d=0,33)$ quanto cromáticos $(d=0,34)$. Analisando o grupo concordante, houve diferença de moderada magnitude entre os métodos nos estímulos acromáticos $(d=0,55)$ e os cromáticos $(d=0,43)$. No grupo discordante, Zulliger e Rorschach tiveram o estímulo cromático $(d=0,24)$ e acromático/vermelho $(d=0,34)$ apresentando fracas magnitudes. Essa diferença de resultados, de modo geral, reflete uma discordância acima do esperado para os dois métodos. Devido às similaridades de Zulliger e Rorschach, a expectativa era que os resultados apresentassem maior similaridade entre os instrumentos (Villemor-Amaral et al., 2016).

Em relação especificamente às pesquisas com amostra depressiva, estudos prévios encontraram algumas variáveis relacionadas à sintomatologia depressiva tanto no Zulliger como no Rorschach. Por exemplo, Villemor-Amaral e Machado (2011) compararam variáveis do Zulliger baseadas no sistema Compreensivo em 27 pacientes depressivos e 27 participantes não clínicos, todos do sexo feminino. Os resultados encontraram-se significativamente aumentados em pacientes depressivos nos indicadores FD+V, Sum-SH, Egocentrismo, $\mathrm{CF}+\mathrm{C}<\mathrm{FC}$, Determinantes mistos e Intelectualização. Quanto aos indicadores Determinantes Mistos CorSH e Sum-SH $>$ FM $+m$ alcançaram uma diferença de magnitude moderada entre os grupos depressivos e não depressivos. No que diz respeito aos indicadores Mor, $\mathrm{S}, \mathrm{Fr}+\mathrm{rF}, \mathrm{COP}$ e Isolamento (também componentes da Constelação de Depressão utilizada pelo SC), não houve diferenças estatísticas significativas.

Ainda no Sistema Compreensivo, Franco e Villemor-Amaral (2012) objetivaram estudar o Zulliger e as Constelações do Rorschach. Dentre as Constelações estudadas estavam presentes a DEPI, conjunto associado a indicadores de depressão. A amostra foi dividida em dois grupos, não pacientes, com 95 sujeitos, e pacientes psiquiátricos com 46, sendo que destes, 18 eram depressivos. Apenas o indicador $\mathrm{AB}$ mostrou-se sensível para 
diferenciar pacientes depressivos de um grupo não clínico. Esses resultados indicam que ainda há carência de estudos com grupo de depressivos e que são necessárias novas pesquisas para que seja possível entender, realmente, quais variáveis são mais sensíveis e, consequentemente, melhor avaliam a depressão.

Uma possível explicação para divergências nas correlações entre ambos os instrumentos apontada por outros pesquisadores, mas ainda não testada empiricamente é a variabilidade no número de respostas dadas (Cárpio \& Lugón, 2011; Villemor-Amaral \& Cardoso, 2012; Villemor-Amaral et al., 2016). Essa variável poderia, por exemplo, aumentar ou diminuir as chances de determinados conteúdos serem apresentados, interferindo nas correlações. Dado que pacientes depressivos, assim como outros grupos psiquiátricos, tendem a produzir menos repostas quando comparado ao grupo de não pacientes (Villemor-Amaral \& Primi, 2009), uma forma de tentar minimizar o aparecimento de protocolos muito curtos e procurar melhorar as evidências de validade do Zulliger ao compará-lo com o Rorschach é o controle do número de respostas, denominado R-otimizado, procedimento já utilizado com sucesso no Rorschach (R-PAS) (Meyer et al., 2017; Pianowski, Meyer, \& Villemor-Amaral, 2016a, 2016b; Viglione et al., 2015). Esse procedimento não se limita a população psiquiátrica, podendo também ser testado em amostras gerais, a fim de verificar seu funcionamento diante de protocolos muito extensos ou muito reduzidos.

A aplicação R-otimizada é uma das particularidades da administração de um novo sistema para o teste de Rorschach, o R-PAS, que consiste em limitar um intervalo de duas a três respostas por cartão (as especificidades das instruções do R-PAS estão na seção Método). O objetivo desse tipo de aplicação é evitar que protocolos fiquem excessivamente longos ou curtos e afetem os resultados da avaliação (Meyer et al., 2017). Especificamente no Zulliger, Villemor-Amaral et al. (2016) verificaram que protocolos com aproximadamente seis respostas (em média duas por cartão) são demasiadamente instáveis e pouco contribuem para interpretabilidade do teste. Dessa forma, adotar os mesmos parâmetros do R-PAS (resultando em protocolos próximos de seis a nove respostas) continuaria muito breve e pouco confiável.

Villemor-Amaral et al. (2016) sugeriram que um protocolo interpretável deveria ter, no mínimo, em torno de nove respostas (aproximadamente três por cartão). Em pesquisas anteriores com o Zulliger (VillemorAmaral et al., 2016; Villemor-Amaral \& Primi, 2009), a média de respostas foi por volta de oito a nove (com desvio padrão variando entre 3 a 3,6), ficando marginal ao mínimo necessário e podendo comprometer a análise de algumas variáveis. Tendo isso em consideração, objetivou-se desenvolver um método que forçasse um número mínimo de respostas, instruindo o participante a apresentar, ao menos, três por cartão, esperando-se, com isso, um mínimo próximo a nove. Por fim, levando em consideração os valores de desvio padrão das amostras referenciadas, optou-se por testar empiricamente um limite máximo de até aproximadamente dois desvios padrão acima da média, ou seja, entre 14 a 16 respostas ao total (cinco por cartão).

Assim, pretendeu-se testar empiricamente o funcionamento do teste, inserindo instrução para que o participante dê três a cinco respostas por cartão. Dessa forma, seriam obtidos protocolos em que se esperam ao menos nove respostas (conforme indicação de Villemor-Amaral et al., 2016), mas evitar-se-ia protocolos demasiadamente longos ao limitar até 15 respostas por cartão (aproximadamente dois desvios padrão). Ressalta-se que essa testagem é empírica e inédita, ou seja, trata-se de uma exploração da estabilidade dos dados a partir da instrução referida.

Dada a similaridade entre o Zulliger e Rorschach e, especificamente neste estudo, com a hipótese de que protocolos muito curtos possam afetar a estabilidade e qualidade dos dados encontrados, esta pesquisa tem como objetivo buscar evidências de validade convergente para Zulliger aplicação R-otimizada por meio das correlações com o Rorschach nas variáveis relacionadas à depressão. Serão consideradas para análise as variáveis elencadas como relacionadas à depressão, a saber: Determinantes de sombreado e cor acromática (FV, VF, V, FY, YF, Y, FC', C'F, C'); Determinante de reflexo (Fr, rF); Determinantes mistos de sombreado; Códigos especiais (COP, MOR, AB); Uso de espaço em branco; Respostas pares; e Conteúdos (Natureza, Botânica, Artístico, Paisagem, Nuvem). Também serão analisadas as seguintes fórmulas: Índice de Egocentrismo, Coeficiente de Afetividade, Índice de Isolamento, Determinantes Mistos de Cor e Sombreado e Índice de Intelectualização (Villemor-Amaral \& Primi, 2009). Hipotetiza-se que, ao controlar o número de respostas produzidas no Zulliger, as correlações entre ambos os instrumentos aumentarão nas variáveis relacionadas à sintomatologia depressiva.

\section{Método}

\section{Participantes}

A amostra deste estudo contou com 39 participantes com idades entre 18 anos a 62 anos $(M=34,90$; $D P=11,54)$, a maioria do sexo feminino $(83,2 \%)$, com escolaridade de ensino médio completo $(43,6 \%)$ e ensino superior incompleto $(28,2 \%)$. Todos os participantes tinham como diagnóstico primário depressão recorrente e estavam em tratamento no Centro de Atenção Psicossocial (CAPS) e na Estratégia da Saúde da Família (ESF) de uma cidade do interior de Minas Gerais; 10\% da amostra fazia tratamento psiquiátrico no Ambulatório do Hospital das Clínicas (AHC) de uma cidade do interior paranaense. Como critérios de inclusão, o usuário deveria ter idade mínima de 18 anos, apresentar em seu 
prontuário o número CID referente à depressão recorrente (F33), além de manifestar sintomatologia depressiva igual ou superior à classificação "leve" na Escala Baptista de Depressão (EBADEP-A).

\section{Instrumentos}

\section{Escala Baptista de Depressão - Adulto}

A EBADEP-A é um inventário de autorrelato usado para rastrear a sintomatologia depressiva em amostras psiquiátricas e não psiquiátricas. É composto por 45 itens, cada um contendo duas frases opostas em termos de sintomatologia depressiva. O respondente deve pontuar, em uma escala de 4 pontos, o mais próximo da afirmativa correspondente a como se sentiu nas últimas duas semanas. Trata-se de uma escala que apresenta um escore geral que varia de zero a 135 , sendo que, quanto menor a pontuação menor a sintomatologia das depressões. Para a presente pesquisa, foi adotada pontuação mínima igual a 60, correspondente a classificação "leve". O manual traz uma divisão teórica da escala em sete categorias de itens: Ansiedade (relacionada a pensamentos hipocondríacos), Irritabilidade (relativa à sensação de estar irritável), Motores (referente à agitação ou à lentidão motora), Vegetativos ou Somáticos (indicativo de fadiga, perda de energia, alteração de sono, apetite, peso e libido), Cognitivos (alusivo a pensamentos de desamparo, indecisão, redução da concentração, autocrítica exacerbada, ideação suicida e culpa), Humor (relativo a humor deprimido, choro, anedonia, autoestima rebaixada, falta de perspectiva no presente) e Sociais (referente à incapacidade, queda de produtividade, carência/dependência, inadequação, inutilidade, esquiva de situações sociais). A escala apresenta consistência interna de $\alpha=0,95$ (Baptista, 2012) e, na presente amostra, $\alpha=0,89$. Esse instrumento foi utilizado com objetivo de triar os participantes, selecionando apenas aqueles que apresentassem sintomatologia depressiva, constituindo assim mais uma confirmação do critério de inclusão, para além do que está registrado nos prontuários dos participantes.

\section{Método de Zulliger}

Composto por três cartões com manchas de tintas, sendo uma acromática, uma cromática com inserção somente da cor vermelha e outra totalmente cromática. A aplicação consiste em duas fases consecutivas: inicialmente são apresentadas as pranchas, uma a uma, e solicita-se que o examinando diga com o que as manchas se parecem. Posteriormente, as pranchas são reapresentadas, dessa vez para que o sujeito indique a localização das respostas dadas na primeira fase e o que fez parecer com aquilo que foi dito. Os estudos de fidedignidade do teste, por meio do método teste e reteste e confiabilidade entre avaliadores, indicaram valor acima de $r=0,70$ para concordância entre juízes e correlação acima de $r=0,60$ entre primeira e a segunda aplicação, indicando uma associação alta (Villemor-Amaral \& Primi, 2009). Atendendo à proposta de controlar o número de respostas (R), foi solicitado que o participante desse de três a cinco respostas por prancha. Essa aplicação objetiva principalmente forçar um número médio de respostas maior que o habitual, além de diminuir a variabilidade entre os protocolos, evitando que fossem muito curtos ou muito longos. $\mathrm{O}$ número de repostas por cartão aqui proposto é empírico e teve como finalidade testar a sugestão proposta por Villemor-Amaral et al., (2016).

\section{Método de Rorschach}

Consiste em 10 pranchas com manchas de tintas das quais cinco são acromáticas e cinco cromáticas. A aplicação é dividida em duas etapas: fase de resposta e fase de esclarecimento. Na primeira, é solicitado que o sujeito diga com o que as manchas de tinta se parecem. Na segunda etapa, solicita-se que o indivíduo indique a localização e elementos da mancha que contribuíram para percepção do que foi dito na fase de resposta (Meyer et al., 2017).

Com o objetivo de controlar o número de respostas (R), as instruções de aplicação foram baseadas nas contribuições do R-PAS (Meyer et al., 2017). Nesse sistema, é solicitado ao testando que dê duas, talvez três respostas por prancha. Para a codificação e análises das respostas, foi usado o Sistema Compreensivo com intuito de possibilitar comparações com as variáveis do Zulliger que, no momento, está disponível no Brasil. Dessa forma, o Sistema Compreensivo é o único comum a ambos os instrumentos e, com a finalidade de evitar codificações ou comparações equivocadas devido às divergências de sistemas, optou-se por um sistema já pesquisado e utilizado com ambos os testes.

\section{Procedimentos}

\section{Procedimentos de Coleta de Dados}

Os dados foram coletados de forma individual em uma única sessão. $O$ primeiro instrumento aplicado foi a EBADEP-A com finalidade de rastrear sintomas depressivos nos usuários. Apenas aqueles que atingiram classificação leve a severa eram convidados a responder aos demais instrumentos. Com o objetivo de minimizar o efeito de uma técnica sobre a outra, a aplicação dos testes de Zulliger e Rorschach foi alternada, ou seja, ora o Zulliger era apresentado primeiro e logo após o Rorschach ora o inverso. Além disso, primeiramente a fase de associação de ambas as tarefas era realizada para, em seguida, iniciar-se a fase de inquérito. Para o teste de Zulliger, foi solicitado de três a cinco respostas por cartão e para o Rorschach de duas, talvez três respostas.

\section{Procedimentos de Análise de Dados}

Para avaliar a precisão de codificação, $25 \%$ dos protocolos foram submetidos à análise de três juízes treinados no Sistema Compreensivo. Esse procedimento é 
recomendado para maior fidedignidade dos resultados encontrados. Para isso, foi utilizado o Coeficiente de Correlação Intraclasse (ICC). Os resultados foram analisados conforme as diretrizes de Cicchetti (1994) em que valores abaixo de 0,40 são classificados como ruins; de 0,40 a 0,59 regulares; de 0,60 a 0,74 bons; e a partir de 0,75 são excelentes. Para alcançar o objetivo do estudo, foi realizada correlação de Person entre os códigos relacionados à depressão no Zulliger e no Rorschach, além de correlações do Zulliger com EBADEP-A, considerando a classificação proposta por Cohen (1992) em que valores ao redor de 0,10 são avaliados como fracos; ao redor de 0,30 são considerados moderados; e ao redor de 0,50 são classificados como fortes. Serão significativos os valores de $\mathrm{p} \leq 0,05$. Para as análises, foi utilizado o programa estatístico Statistical Package for the Social Sciences 20.0I.

\section{Considerações Éticas}

O estudo seguiu todos os dispositivos da Resolução do Conselho Nacional de Saúde n. ${ }^{\circ} 196$ e seus complementos, tendo sido submetido à apreciação de um Comitê de Ética em Pesquisas. Após aprovação do estudo pelo Comitê de Ética em Pesquisas da Universidade
São Francisco (CAAE: 78320917.9.0000.5514; Parecer n. ${ }^{\circ}$ 2409144) foi realizado o convite via contato telefônico a pacientes designados pelos referidos centros de tratamento que atendiam aos critérios de inclusão já citados. Os participantes foram informados sobre os objetivos da pesquisa e procedimentos da coleta e, após anuência, assinaram o Termo de Consentimento Livre e Esclarecido.

\section{Resultados}

Os valores do Coeficiente de Correlação Intraclasse (ICC) para o Rorschach variaram entre regular $(0,48) \mathrm{e}$ excelente (1) e, no Zulliger, entre regular $(0,59)$ e excelente (1), como pode ser visto na Tabela 1. As discordâncias foram verificadas e a dupla de juízes, ou com o auxílio de um terceiro juiz, definiu qual a melhor codificação para aquela resposta. Em seguida, os demais protocolos foram revisados nas variáveis mais problemáticas identificadas por baixa concordância entre juízes e foram calibrados de modo a melhorar a fidedignidade do banco de dados. Os valores de concordância estão em acordo com o encontrado na literatura (Kivisalu, Lewey, Shaffer, \& Canfield, 2016; Meyer et al., 2017; Pignolo et al., 2017).

Tabela 1

Coeficiente de Correlação Intraclasse (ICC) das Variáveis Relacionadas à Depressão no Zulliger e Rorschach

\begin{tabular}{ccccc}
\hline Códigos & ICC-Rorschach & Classificação $^{a}$ & ICC-Zulliger & Classificação $^{a^{a}}$ \\
\hline C' & 0,81 & Excelente & 0,92 & Excelente $^{\prime}$ \\
Y & 0,89 & Excelente & 0,83 & Excelente \\
V & 0,73 & Bom & 1 & Excelente \\
T & 0,83 & Excelente & 0,91 & Excelente \\
FD & 0,80 & Excelente & 1 & Excelente \\
r & 1 & Excelente & 1 & Excelente \\
S & 0,77 & Excelente & 0,84 & Excelente \\
Art & 1 & Excelente & 1 & Excelente \\
Ay & 0,44 & Regular & 0,75 & Regular \\
Na & 0,88 & Excelente & 0,59 & Excelente \\
Cl & 1 & Excelente & - & Excelente \\
Bt & 0,73 & Bom & 0,94 & Excelente \\
Ls & 0,82 & Excelente & 0,86 & $\mathrm{~b}$ \\
$\mathrm{Ge}$ & 1 & Excelente & $\mathrm{b}$ & $\mathrm{b}$ \\
$\mathrm{AB}$ & 0,98 & Excelente & $\mathrm{b}$ & Excelente \\
$\mathrm{MOR}$ & 0,84 & Excelente & 1 & Bom \\
$\mathrm{COP}$ & 0,50 & Regular & 0,73 & Excelente \\
(2) & 0,86 & Excelente & 0,89 &
\end{tabular}

Nota. ${ }^{a}=$ Classificação segundo Cicchetti (1994); ${ }^{b}=$ variável constante

Os valores médios de respostas por protocolo no Rorschach e Zulliger foram 23,3 (DP=2,96) e 9,9 $(D P=1,63)$, respectivamente. Nessa amostra, o valor médio de respostas no Rorschach seguiu o esperado para aplicação R-otimizada nesse instrumento, entre 18 e 27
(Meyer et al., 2017). No Zulliger, o valor médio de respostas apresentado pela amostra normativa de pacientes foi $M=6,5(D P=2,7)$ (Villemor-Amaral \& Primi, 2009).

Foi realizada correlação de Pearson entre os testes de Zulliger e Rorschach nas variáveis que estão relacionadas 
à depressão e também a correlação dessas variáveis do Zulliger com o escore total e categorias da EBADEP-A, conforme apresentado na Tabela 2. A seleção das variáveis baseou-se na literatura da área (Exner \& Sendín, 1999; Meyer et al., 2017; Mihura, Meyer, Dumitrascu, \& Bombel, 2013; Villemor-Amaral \& Primi, 2009).

Em relação à Categoria Determinantes, nota-se que as correlações entre Zulliger e Rorschach foram significativas e positivas, de magnitudes moderadas e altas, exceto o código $\mathrm{V}$ cuja correlação foi baixa e não estatisticamente significativa. As correlações mais fortes observadas nesse grupo de variáveis foram C', T e Y. Já as respostas reflexo (r) apresentaram correlação moderada entre ambos instrumentos. Em relação a EBADEP-A, a variável $Y$ foi a que apresentou maior número de correlações, seguida de $r$ e $C^{\prime}$.

Tabela 2

Correlação entre a Porcentagem do Total de Respostas das Variáveis Relacionada à Depressão no Zulliger com Variáveis do Rorschach e Categorias da EBADEP-A

\begin{tabular}{|c|c|c|c|c|c|c|c|c|c|c|}
\hline & Códigos & $\begin{array}{l}\text { Va. Equi. } \\
\text { Rorschach }\end{array}$ & Total & Ansi. & Irrita. & Mot. & $\begin{array}{l}\text { Veg. e } \\
\text { Som. }\end{array}$ & Soc. & Hum. & Cog. \\
\hline \multirow[t]{5}{*}{ Determ. } & $C^{\prime}$ & $0,77^{* *}$ & 0,21 & $-0,15$ & 0,18 & $-0,09$ & 0,01 & 0,21 & $0,33^{*}$ & 0,22 \\
\hline & $\mathrm{T}$ & $0,68^{* *}$ & 0,18 & $-0,10$ & $-0,05$ & $-0,01$ & 0,18 & 0,16 & 0,13 & 0,11 \\
\hline & $\mathrm{Y}$ & $0,64^{* *}$ & $-0,32^{*}$ & 0,11 & $-0,15$ & $-0,33^{*}$ & $-0,48^{* *}$ & $-0,17$ & $-0,31$ & $-0,15$ \\
\hline & $r$ & $0,44^{* *}$ & 0,11 & $0,42^{* *}$ & 0,29 & 0,14 & 0,00 & 0,05 & 0,13 & 0,09 \\
\hline & $\mathrm{V}$ & 0,03 & 0,13 & $-0,02$ & 0,25 & $-0,17$ & 0,22 & 0,05 & 0,23 & 0,00 \\
\hline \multirow{8}{*}{$\begin{array}{l}\text { Códigos } \\
\text { Especiais } \\
\text { e Î́ndices }\end{array}$} & COP & $-0,07$ & 0,13 & $-0,02$ & 0,25 & $-0,17$ & 0,22 & 0,05 & 0,23 & 0,00 \\
\hline & $A B$ & 0,11 & 0,01 & $-0,09$ & $-0,33^{*}$ & 0,06 & $-0,07$ & 0,02 & 0,14 & 0,02 \\
\hline & MOR & 0,25 & 0,24 & $-0,08$ & $-0,05$ & 0,05 & 0,04 & 0,22 & 0,23 & 0,28 \\
\hline & Ego & $0,46^{* *}$ & $-0,20$ & 0,03 & $-0,18$ & $-0,24$ & $-0,22$ & $-0,29^{*}$ & 0,00 & $-0,15$ \\
\hline & Isolate & 0,22 & 0,11 & $-0,29$ & 0,21 & $-0,14$ & 0,08 & 0,20 & 0,10 & 0,06 \\
\hline & Intelec & $0,36^{*}$ & 0,30 & $-0,06$ & $-0,22$ & 0,05 & 0,07 & $0,32^{*}$ & $0,35^{*}$ & 0,28 \\
\hline & Afr & 0,01 & 0,29 & $-0,07$ & $-0,06$ & 0,10 & 0,22 & 0,22 & 0,24 & 0,23 \\
\hline & Det. So. & $0,32^{* *}$ & 0,01 & $-0,04$ & 0,14 & $-0,29$ & 0,21 & 0,09 & 0,06 & 0,08 \\
\hline \multirow[t]{6}{*}{ Cont. } & $\mathrm{Na}$ & $-0,17$ & 0,16 & $-0,07$ & 0,18 & $-0,10$ & $-0,06$ & 0,25 & 0,13 & 0,20 \\
\hline & $\mathrm{Cl}$ & 0,28 & 0,06 & $-0,13$ & 0,20 & $-0,01$ & 0,08 & 0,09 & 0,12 & $-0,05$ \\
\hline & $\mathrm{Bt}$ & $0,36^{*}$ & 0,06 & $-0,13$ & 0,20 & $-0,01$ & 0,08 & 0,09 & 0,12 & $-0,05$ \\
\hline & Art & $0,73^{* *}$ & 0,00 & $-0,13$ & $-0,12$ & $-0,26$ & $-0,04$ & 0,05 & 0,00 & 0,03 \\
\hline & Ay & $-0,10$ & $0,43^{* *}$ & 0,13 & 0,06 & 0,06 & 0,30 & $0,35^{*}$ & $0,38^{*}$ & 0,28 \\
\hline & Ls & 0,05 & $-0,24$ & $-0,15$ & $-0,04$ & $-0,27$ & $-0,17$ & $-0,14$ & 0,00 & $-0,24$ \\
\hline \multirow[t]{2}{*}{ Outros } & S & 0,30 & 0,05 & 0,10 & $-0,06$ & 0,15 & $-0,01$ & 0,01 & $-0,08$ & 0,02 \\
\hline & (2) & $0,55^{* *}$ & $-0,28$ & $-0,15$ & $-0,27$ & $-0,34^{*}$ & $-0,25$ &,$- 33^{*}$ & $-0,11$ & $-0,20$ \\
\hline
\end{tabular}

Nota. Determ.=Determinantes; Cont.=Conteúdo; $C^{\prime}=$ soma dos determinantes de cores branca, cinza e preta $\left(C^{\prime}+C^{\prime} F+F C^{\prime}\right)$; $\mathrm{T}=$ soma dos determinantes Textura $(\mathrm{T}+\mathrm{TF}+\mathrm{FT})$; $\mathrm{Y}=$ soma dos determinantes sombreado $(\mathrm{Y}+\mathrm{YF}+\mathrm{FY})$; $\mathrm{r}=\mathrm{soma}$ dos determinantes Reflexo ( $\mathrm{Fr}+\mathrm{rF}) ; \mathrm{V}=$ soma dos determinantes Vista $(\mathrm{V}+\mathrm{VF}+\mathrm{FV})$; $\mathrm{COP}=$ conteúdo de movimento cooperativo; $\mathrm{AB}=\mathrm{conteúdo} \mathrm{abstrato;}$ $\mathrm{MOR}=$ conteúdo mórbido; EGO=índice de egocentrismo; Isolate=índice de isolamento; Intelec=índice de intelectualização; $\mathrm{AFR}=$ coeficiente de afetividade; Det. So.=determinantes mistos de cor e sombreado; $\mathrm{Na=conteúdo} \mathrm{de} \mathrm{natureza;} \mathrm{Cl}=\mathrm{conteúdo} \mathrm{de}$ nuvem; Bt=conteúdo de botânico; Art=conteúdo de arte; Ay=conteúdo de antropologia; Ls=conteúdo de paisagem; S=respostas que inclui a parte em branco do cartão; (2)=resposta par. Va. Equi. Rorschach=variáveis equivalente no Rorschach; Total=Escore Total EBADEP-A; Ansi.=ansiedade; Irrita. = irritabilidade; Mot.=Motor; Veg. E Som.=vegetativos e somáticos; Soc.=sociais; Hum. $=$ humor; Cog. $=$ cognitivos. ${ }^{*} p \leq 0,05 ;{ }^{* *} p \leq 0,01$

As correlações dos códigos especiais, bem como o índice de Isolamento e Coeficiente de Afetividade, não foram estatisticamente significativas. Já os índices de Egocentrismo, de Intelectualização e Determinantes de Sombreado apresentaram correlações positivas, significativas e moderadas com o Rorschach e facetas da EBADEP-A. Na categoria Conteúdo, arte (Art) apresentou maior correlação entre os dois testes de manchas de tinta. As respostas referentes ao conteúdo botânica $(\mathrm{Bt})$ apresentaram correlação significativa positiva e de moderada magnitude, sendo considerado uma boa concordância entre Zulliger e Rorschach nesse código. As respostas par (2) obtiveram valores positivo de forte magnitude. Por fim, no que se refere às respostas que 
utilizam da parte branca da mancha (S) para construir o objeto, houve correlação positiva e de moderada magnitude, esse resultado foi marginalmente significativo. Por fim, antropologia (Ay), que embora não tenha apresentado correlações significativas entre Zulliger e Rorschach, apresentou associação positiva e moderada com três facetas da EBADEP-A.

Em relação às correlações entre EBADEP-A e as variáveis do Zulliger, em síntese, observou-se que houve correlação de 14 códigos com as categorias de itens da escala de depressão sendo que quatro destas foram com itens relacionados à categoria Social e três relacionado a Humor, com valores moderados. A categoria Cognitivo não apresentou correlação com nenhuma variável do Zulliger.

\section{Discussão}

Esse estudo teve como objetivo principal verificar as correlações entre os testes de Zulliger e Rorschach quando controlado o número de respostas em ambos os instrumentos em pacientes depressivos. Os dados representam achados importantes para o teste de Zulliger aplicação R-otimizada. Sua principal contribuição é indicar que, ao controlar empiricamente o número de respostas no Zulliger, a capacidade de captar aspectos relacionados à sintomatologia depressiva melhora em relação a estudos prévios com amostra depressiva (Villemor-Amaral \& Machado, 2011) e em comparação entre variáveis do Rorschach e Zulliger (Cárpio \& Lugón, 2011).

Um primeiro ponto a ser ressaltado é que, ao controlar o número de respostas no Zulliger, houve ligeiro aumento da média de respostas e importante diminuição na variabilidade delas (desvio padrão), quando comparada à amostra normativa de pacientes. Isso indica eficiência da aplicação R-otimizada em melhorar esse ponto, aspecto já atingido no sistema R-PAS (Meyer, et al., 2017). Esse dado já evidencia a validade do procedimento em questão para aumentar e controlar a variação de respostas.

Especificamente em relação às correlações observadas, os quatro determinantes aqui destacados estão ligados à sintomatologia tipicamente depressiva (Meyer et al., 2017) e, por isso, eram esperadas correlações positivas, de moderada à forte magnitude, entre os instrumentos Zulliger e Rorschach. O uso da cor acromática (C') está relacionado à tentativa de contenção emocional de afetos desprazerosos, além de atitude entristecida diante dos eventos da vida. $\mathrm{O}$ código $\mathrm{T}$ refere-se à necessidade afetiva e a busca por contato, além do mal-estar experimentado com sentimento de solidão e abandono. O determinante $\mathrm{Y}$, pode indicar atenção e capacidade de percepção de estados ansiosos frente a situações conflituosas e estressoras além de tensões afetivas que comprometem o funcionamento normal do indivíduo. Respostas com reflexo (r) podem representar uma maior dificuldade na tomada de decisões ocasionada por um processo de maturidade pessoal deficitário (Exner \& Sendín, 1999; Meyer et al., 2012; Mihura, et al., 2013; Villemor-Amaral \& Primi, 2009).

A observação dessas correlações com o Rorschach indica que o controle do número de respostas melhora a previsão de que tais variáveis, relacionadas à depressão, aparecem aumentadas nos protocolos de Zulliger de pessoas que apresentam esse diagnóstico (Villemor-Amaral \& Machado, 2011). Embora fosse esperado que o código $\mathrm{V}$ também apresentasse correlações altas, hipotetiza-se que isso não foi verificado devido à baixa frequência dessa variável na presente amostra. Dessa forma, em novos estudos, deve-se atentar para essa questão e analisar se realmente trata-se de uma especificidade ou alguma variável relacionada ao R-otimizado.

Além de correlação entre ambos os testes de mancha de tinta, pode-se verificar consistências de correlação também com a escala de sintomatologia depressiva com magnitudes moderadas. Dessa forma, aumento de sintomatologia relacionada a humor deprimido e anedonia (categoria Humor) esteve associado ao aumento de C'. A interpretação de ambas as variáveis é concordante, o que implica numa evidência de que o controle de respostas no Zulliger possibilitou captar o aumento de C' condizente com a percepção de sintomas de humor deprimido. A associação positiva entre respostas reflexo (r) e aumento de sintomatologia da categoria Ansiedade podem ser condizentes com maior dificuldade em autopercepção, conforme observado em estudo de caso conduzido por Rovinski, Nascimento e Scortegagna (2015) utilizando o Rorschach no SC. Quanto ao Y, pode-se entender que as correlações negativas estão relacionadas ao direcionamento do sujeito para questões mais intrínsecas do que para uma angústia mobilizada por estímulos circunstanciais.

Dentre os códigos especiais e índices, o Egocentrismo apresentou a maior correlação entre Zulliger e Rorschach. Ele é usado para avaliar o grau em que a pessoa está preocupada com suas próprias questões e o quanto está voltada para satisfação de suas próprias necessidades (Exner \& Sendín, 1999; Villemor-Amaral \& Primi, 2009). Nos estudos de Villemor-Amaral e Machado (2011) e Hisatugo e Yazigi (2014) sobre os indicadores de depressão no Zulliger e Rorschach, respectivamente, o índice de Egocentrismo foi sensível na diferenciação do grupo de depressivos e não pacientes, apresentando-se aumentado no primeiro, corroborando a importância do egocentrismo, ou seja, o quanto a pessoa está voltada para suas próprias questões nessa psicopatologia. Houve correlação negativa entre essa variável e a categoria Social que está relacionada à sensação de incapacidade, inadequação e esquiva social. Isso leva à hipótese de que o sujeito mais voltado às questões intrapessoais possa não estar atento ao que acontece ao redor em termos interpessoais, o que pode ter contribuído para o achado neste estudo. Ainda assim, é 
necessário rever se o mesmo padrão ocorre em outras amostras e investigar questões relacionadas à percepção social autorrelatada.

O índice de Intelectualização, que se refere aos esforços cognitivos da pessoa para neutralizar afetos, apresentou correlações moderadas entre Zulliger e Rorschach. Esse indicador também apresentou aumento no estudo com Rorschach de Hisatugo e Yagizi (2014) e com Zulliger de Villemor-Amaral e Machado (2011), indicando ser uma importante variável discriminadora de pacientes depressivos em ambos os instrumentos. O índice de intelectualização também apresentou correlações positivas com as categorias Social e Humor da EBADEP-A, o que pode estar relacionado a dificuldades emocionais. Nesse caso, hipotetiza-se que os sujeitos foram hábeis em identificar sentimentos negativos (aqui representados pelas categorias Social e Humor) e como um mecanismo para evitar tais afetos, podem usar da cognição para tentar inibir tais sensações.

Os Determinantes de Sombreado, que dizem respeito às experiências emocionais dolorosas (Exner \& Sendín,1999; Villemor-Amaral \& Primi, 2009), apresentaram também correlação positiva, moderada e estatisticamente significativa entre Zulliger e Rorschach. Dessa forma, sua correlação aumentada entre os instrumentos, em uma amostra clínica como a presente, demonstra evidência de validade para o tipo de controle aqui empregado.

Em relação aos códigos de Conteúdo, a melhor correlação, Arte (Art), contrastou com o encontrado no estudo de Cárpio e Lugán (2011) que verificou correlações fracas nesse código. Analisando os dados, é possível inferir que esse código apresentou melhores correlações entre Zulliger e Rorschach quando um número de respostas foi controlado no Zulliger, demonstrando benefícios da aplicação R-otimizada, proporcionando evidências de validade. Já o conteúdo Bt apresentou dados muito próximos aos encontrados por Cárpio e Lugán (2011) que também encontraram correlação positiva, de moderada magnitude e estatisticamente significativa $(r=0,35 ; p<0,01)$. Esses resultados demonstram que, mesmo que a diferença seja pequena, não houve alteração negativa entre os estudos, com os dados mantendo-se estáveis independentemente do modo de aplicação. Por fim, o conteúdo Ay não apresentou correlações entre os testes de mancha de tinta. Porém, houve três correlações positivas com a EBADEP-A (escore total e categorias Social e Humor). Assim como a intelectualização, Ay está associado a constrições e tentativa de neutralizar estados emocionais, o que caminha na mesma direção da hipótese para as correlações com ambas categorias da EBADEP-A.

A correlação entre o Zulliger e Rorschach apresentou melhores valores para respostas pares (2) quando comparado aos resultados encontrados por Cárpio e Lugón $(2011 ; r=0,52$ e $p=0,01)$. Ainda que a diferença entre os estudos seja pequena nas respostas pares, pode-se entender como um bom indicador de validade o Zulliger aplicação R-otimizada no que diz respeito a essa categoria de resposta. Esse indicador está correlacionado negativamente com as categorias de sintomatologia Motora e Social da EBADEP-A. A direção negativa das correlações indica que os sujeitos dessa amostra tenderam a apresentar dificuldades na percepção e interação com o outro. As respostas S estão relacionadas com um comportamento mais opositor e negativista (Exner \& Sendín,1999; Villemor-Amaral \& Primi, 2009). Na pesquisa por Cárpio e Lugón (2011), a correlação encontrada foi de fraca magnitude $(r=0,18)$ indicando que, com a aplicação R-otimizada, esse tipo de resposta foi afetado demonstrando melhorias na relação entre os testes de mancha de tinta.

Algumas correlações esperadas entre os testes de manchas de tintas não foram encontradas, como é o caso do código especial MOR. Este se refere a respostas com conteúdo mórbido, pessimista e sentimentos disfóricos. Sua presença pode indicar problemas com sua autoimagem e, quando aumentado em um protocolo, refere-se desesperança com aspectos gerais da vida (Exner \& Sendín, 1999). Embora esse código seja indicado como de grande importância em quadros depressivos (Meyer et al., 2017) outros estudos também não encontraram evidências de discriminação entre amostra depressiva e população geral (Hisatugo \& Yazigi, 2014; VillemorAmaral \& Machado, 2011), reforçando a ideia de que mais estudos precisam ser realizados para verificar variáveis influenciadoras de tais resultados. De forma geral, ressalta-se que o tamanho da amostra deste estudo $(n=39)$ possa ter impactado nos resultados e novos estudos com amostra maiores auxiliarão na verificação da replicabilidade dos dados, sendo um ponto importante a ser investigado em estudos futuros.

É possível perceber que houve concordância entre os testes de Zulliger e Rorschach, principalmente quanto aos Determinantes relacionados à depressão; dos cinco analisados, três apresentaram correlações fortes e uma moderada, dados considerados importantes, pois demonstram concordância entre os dois instrumentos. Quanto aos códigos especiais, não houve correlações estatisticamente significativas, o que pode ser explicado pela média em que esses códigos apareceram nos protocolos. Quanto aos conteúdos analisados, apenas dois apresentaram correlações estatisticamente significativas. No que se refere a respostas com espaço em branco (S) e respostas par (2), ambos demonstraram boa concordância entre os instrumentos.

Em relação às correlações observadas entre Zulliger e EBADEP-A, embora fosse pouco esperada entre instrumentos de performance e autorrelato (Campbell \& Fiske, 1959; Mihura, 2012), foram analisadas relações condizentes com as interpretações das variáveis em questão. A maioria das correlações são referentes a 
indicadores de percepção e formas de lidar com afetos negativos, o que indica que, nessa amostra, a sintomatologia percebida pelos pacientes é condizente com as variáveis selecionadas para análise, indicando evidência de validade complementar para o Zulliger R-Otimizado. Estudos futuros poderiam investigar essas relações com outras sintomatologias.

A hipótese inicial apresentada no presente estudo de que variáveis relacionadas à depressão teriam melhores correlações entre Zulliger e Rorschach quando comparado à literatura foi parcialmente confirmada. Compreende-se, porém, como já apontado, que, por mais que possuam características em comum, Zulliger e Rorschach são testes com diferenças, cada qual com suas particularidades, como o número de cartões e as proporções de cartão acromático, cromático e acromático com inserção do vermelho, podendo estimular respostas diferentes (Mahmood, 1990; Mattlar et al., 1990; Zdunic, 1999). Seguindo a proposta do R-PAS de controlar o número de respostas como uma forma de melhorar os indicadores dos testes, observou-se que o Zulliger provavelmente se beneficia desse tipo de controle. Algumas correlações entre os instrumentos melhoraram quando comparadascom a literatura.

Uma importante limitação deste estudo foi o número de participantes avaliados. Embora o tamanho amostral supere pesquisas anteriores, hipotetiza-se que uma amostra maior poderia contribuir para maiores esclarecimentos quanto às correlações. Para futuras pesquisas que almejem verificar o impacto do número de respostas sobre as variáveis do Zulliger, considera-se válida a variação da amostra, contendo pessoas com e sem outras sintomatologias condizentes com psicopatologias. $\mathrm{O}$ controle de respostas aqui imposto foi empírico, ou seja, foi feita uma testagem de qual melhor intervalo de resposta traria melhores resultados para o Zulliger, baseado em especial na sugestão proposta por Villemor-Amaral et al., (2016) e amostra normativa (Villemor-Amaral \& Primi, 2009). Dessa forma, sugere-se que, em estudos futuros, outros arranjos possam ser testados, visando o número ótimo de respostas. Outra possibilidade seria procurar evidências de validade correlacionando diferentes amostras do Zulliger com aplicação tradicional e aplicação R-otimizada, comparando qual dos modelos de aplicação alcançam melhores resultados.

\section{Referências}

American Psychiatric Association. (2014). Manual diagnóstico e estatístico de transtornos mentais (DSM-5). Porto Alegre: Artmed.

Baptista, M. N. (2012). Manual técnico da Escala Baptista de Depressão em adultos (EBADEP-A). São Paulo: Vetor.

Biasi, F. C., \& Villemor-Amaral, A. E. D. (2016). Evidências de validade do Zulliger - SC para avaliação do relacionamento interpessoal de crianças. Psico, 47(1), 13-23. doi: 10.15448/1980-8623.2016.1.19990

Campbell, D. T., \& Fiske, D. W. (1959). Convergent and discriminant validation by the multitrait-multimethod matrix. Psychological Bulletin, 56(2), 81-105.

Cárpio, S. R., \& Lugón, M. C. (2011). Validación del sistema comprehensivo de Exner en el test de Zulliger: Validation of the Exner comprehensive system in the Zulliger test. Persona, 14, 145-158. Recuperado de http://www.redalyc.org/html/1471/147122650006/

Cicchetti, D. V. (1994). Guidelines, criteria, and rules of thumb for evaluating normed and standardized assessment instruments in psychology. Psychological Assessment, 6(4), 284-290. doi: 10.1037/1040-3590.6.4.284

Cohen, J. (1992). Statistical power analysis. Current Directions in Psychological Science, 1(3), 98-101. Recuperado de https://goo.gl/5XUAy3

Exner, J. E., \& Sendín, C. (1999). Manual de interpretação do Rorschach para o sistema compreensivo. São Paulo: Casa do Psicólogo.

Franco, R. R. C., \& Villemor-Amaral, A. E. (2012). O Zulliger e as constelações do Rorschach no Sistema Compreensivo. Avaliação Psicológica, 11(1), 141-152. Recuperado de https://goo.gl/CqqM3v

Grazziotin, J. B. D, \& Scortegagna, S. A. (2016). Revisão de pesquisas brasileiras sobre o Teste de Zulliger publicadas em artigos. Avaliação Psicológica, 15(2), 227-235. Recuperado de https://goo.gl/XMJaSE

Hisatugo, C. L. C., \& Yazigi, L. (2014). Estudo exploratório com indivíduos com depressão por meio do Rorschach, Sistema Compreensivo. Avaliação Psicológica, 13(2), 157-166. Recuperado de https://goo.gl/UQZVHX

Kivisalu, T. M., Lewey, J. H., Shaffer, T. W., \& Canfield, M. L. (2016). An investigation of interrater reliability for the Rorschach Performance Assessment System (R-PAS) in a nonpatient U. S. sample. Journal of Personality Assessment, 98(4), 382-390. doi: 10.1080/ 00223891.2015 .1118380

Mahmood, Z. (1990). The Zulliger test: Its past and future. British Journal of Projective Psychology, 35(2), 2-16.

Mattlar, C. E., Sandahl, C., Lindberg, S., \& Lehtinen, V. (1990). Methodological issues associated with the application of the comprehensive system when analysing the Zulliger, and the structural resemblance between the Zulliger and the Rorschach. British Journal of Projective Psychology. 35(2), 17-27.

Meyer, G. J. (2017). What Rorschach performance can add to assessing and understanding personality. International Journal of Personality Psychology, 3(1), 36-49. Recuperado de https://goo.gl/QHqg1C

Meyer, G. J, Viglione, D. J., Mihura, J. L., Erard, R. E., \& Erdberg, R. (2017). Rorschach sistema de avaliação por desempenho manual de aplicação codificação e interpretação e manual técnico. Tradução Silva, D. R. \& Miguel, F. K. $1^{\circ}$ ed. São Paulo: Hogrefe.

Mihura, J. L. (2012). The necessity of multiple test methods in conducting assessments: The role of the Rorschach and self-report. Psychological Injury and Law, 5(2), 97-106. doi: 10.1007/s12207-012-9132-9

Mihura, J. L., \& Graceffo, R. A. (2014). Multimethod assessment and treatment planning. In C. J. Hopwood \& R. F. Bornstein (Eds.), Multimethod clinical assessment (pp. 285-318). New York: The Guilford Press. 
Mihura, J. L., Meyer, G. J., Dumitrascu, N., \& Bombel, G. (2013). The validity of individual Rorschach variables: Systematic reviews and meta-analyses of the comprehensive system. Psychological Bulletin, 139(3). 548-605. doi: 10.1037/a0029406

Organização Mundial de Saúde, OMS (2017). Depression. Recuperado de http://www.who.int/mediacentre/factsheets/fs369/en/

Organização Mundial de Saúde, OMS (1993). Classificação Estatística Internacional de Doenças e Problemas Relacionados à Saúde - CID-10. Porto Alegre: Artmed

Pianowski, G., Meyer, G. J., \& Villemor-Amaral, A. E. (2016a) The impact of R-optimized administration modeling procedures on brazilian normative reference values for Rorschach scores. Journal of Personality Assessment, 98(4), 408-418. doi: 10.1080/00223891.2016.1148701

Pianowski, G., Meyer, G. J., \& Villemor-Amaral, A. E. (2016b). Potential projective material on the Rorschach: Comparing Comprehensive System protocols to their modeled r-optimized administration counterparts. Journal of Personality Assessment, 98(4), 398-407. doi: 10.1080/00223891.2016.1147451

Pianowski, G., Villemor-Amaral, A. E., Yazigi, L., Nascimento, R. S. G. F., \& Miguel, F. K. (2017). Apêndice à edição brasileira do R-PAS. Em G. J., Meyer, D. J., Viglione, J. L., Mihura, R. E., P. Erard, Erdberg, (Eds.). R-PAS Sistema de Avaliação por Performance no Rorschach: Manual de aplicação, codificação e interpretação e manual técnico. 1ed. São Paulo: Hogrefe.

Pignolo. C., Giromini. L., Ando. A., Ghirardello. D., Di Girolamo. M., Ales. F., \& Zennaro. A. (2017). An interrater reliability study of Rorschach Performance Assessment System (R-PAS) raw and complexity-adjusted scores. Journal of Personality Assessment, 99(6), 619625. doi: 10.1080/00223891.2017.1296844

Rovinski, S. L. R., Nascimento, R. S. G. F., \& Scortegagna, S. A. (2015). Respostas de reflexo no Rorschach em distintos níveis de adaptação psicossocial. Avaliação Psicológica, 14(2), 243-252

Vaz, C. E., \& Alchieri, J. C. (2016). Z-teste: Coletivo e individual. Técnica de Zulliger. São Paulo: Hogrefe.

Viglione, D. J., Meyer, G. J., Jordan, R. J., Converse, G. L., Evans, J., MacDermott, D., \& Moore, R. (2015). Developing an alternative Rorschach administration method to optimize the number of responses and enhance clinical inferences. Clinical Psychology E Psychotherapy, 22(6), 546-558. doi: 10.1002/cpp.1913

Villemor-Amaral, A. E., \& Cardoso, L. (2012). Validade convergente do tipo de vivência (EB) no Teste de Zulliger/SC. Psico PUCRS, 43(1), 109-115. Recuperado de https://goo.gl/CdqYHV

Villemor-Amaral, A. E., \& Primi, R. (2009). Teste de Zulliger no sistema Compreensivo ZSC: Forma Individual. São Paulo. SP: Casa do Psicólogo.

Villemor-Amaral, A. E., Pianowski, G., \& Carvalho, L, F. (2016). Issues about color, human movement and number of responses in the Zulliger test. Rorschachiana: Journal of the international society for the Rorschach. 37(2), 95-113. doi: 10.1027/1192-5604/a000068

Villemor-Amaral, A. E., \& Machado, M. A. S. (2011). Indicadores de depressão do Zulliger no Sistema Compreensivo (ZSC). Paidéia, 21(48), 21-27. doi: 10.1590/S0103- 863X2011000100004

Weiner, I. B., \& Greene, L. R., (2017), Handbook of Personality Assessment. 2ed. New Jersey: John Wiley \& Sons, Inc.

Zdunic, A. L. (1999). El Teste de Zulliger en la evaluación de personal: Aportes del Sistema Comprehensivo de Exner. Argentina: Paidós.

\section{Sobre os autores}

André Pereira Gonçalves é psicólogo, especialista em Avaliação Psicológica (2017) Mestre e Doutorando em Psicologia com ênfase em avaliação psicológica pela Universidade de São Francisco e membro do Laboratório de Avaliação Psicológica em Saúde Mental (LAPSaM).

Ana Carolina Zuanazzi Fernandes é psicóloga, especialista em Neuropsicologia e Mestrado em Psicologia Clínica pela Universidade de São Paulo (2015). Atualmente é doutoranda pela Universidade São Francisco. É proficiente em Administração e Codificação pelo RorschachR-PAS.

Anna Elisa de Villemor-Amaral é Mestre e Doutor em Ciências pela Universidade Federal de São Paulo (UNIFESP/EPM) e pósdoutorado na Universidade da Savoia/ França. Docente do Programa de Pós-graduação stricto sensu em Psicologia da Universidade São Francisco. Bolsista Produtividade CNPq. 